\title{
Statistical analysis of near-field photoluminescence spectra of single ultrathin layers of $\mathrm{CdSe} / \mathrm{ZnSe}$
}

\author{
G. von Freymann, ${ }^{\text {a) }}$ E. Kurtz, C. Klingshirn, and M. Wegener \\ Institut für Angewandte Physik, Universität Karlsruhe (TH), 76128 Karlsruhe, Germany
}

(Received 13 March 2000; accepted for publication 26 May 2000)

\begin{abstract}
The statistical analysis of thousands of near-field photoluminescence spectra of single ultrathin CdSe layers at $20 \mathrm{~K}$ exhibits a strong positive correlation peak around $20 \mathrm{meV}$ energy with a width of $5 \mathrm{meV}$. Our data are consistent with individual spectra which consist of sets of many pairs of lines. In each pair, the two lines must have comparable strength. We speculate about the origin of these pairs. (C) 2000 American Institute of Physics. [S0003-6951(00)03229-0]
\end{abstract}

Epitaxially grown, thin semiconductor layers often lead to a series of sharp photoluminescence lines in near-field or microphotoluminescence experiments. ${ }^{1-6}$ These individual spectra can exhibit many tens or even hundreds of individual lines and usually look like a random distribution at first sight. What can be learned from this type of spectra? This question was first investigated theoretically in Refs. 7 and 8 for photoluminescence of excitons which move in a truly random potential, i.e., a potential which in itself shows no correlations. They proposed to measure a set of individual spectra, calculate the individual spectral autocorrelation functions, and average over many such autocorrelations. In this fashion, they demonstrated that one can hope to observe correlations in the energy spectrum. In their case, they found the socalled level repulsion, i.e., a negative correlation close to the zero energy difference, which is known from random matrix theory. It is clear that correlations in the spectrum can also arise from correlations in the potential, i.e., from a potential which is not just noise. This would lead to positive correlations.

In this letter, we first modify and extend the proposal ${ }^{7,8}$ in order to make it suitable for measured spectra. This complements recent experiments using time-resolved secondary emission. ${ }^{9}$ As an example, we investigate large sets of near-field photoluminescence spectra of single ultrathin CdSe layers clad between ZnSe barriers.

For the optical experiments, we frequency double about $1 \mathrm{~W}$ power at a $800 \mathrm{~nm}$ wavelength from a continuous-wave Ti:sapphire laser in a 2-mm-thick BBO crystal to obtain about $3 \mu \mathrm{W}$ power at a $400 \mathrm{~nm}$ wavelength $(3.10 \mathrm{eV}$ photon energy). 200-300 nW are effectively coupled into an optical fiber. The light propagates towards a nanometer-scale apex which is formed by a two-step selective etching process. Electron micrographs of identical fiber tips have been shown in Ref. 10. The photoluminescence is collected with the same fiber and sent into a $0.5 \mathrm{~m}$ grating spectrometer (1800 lines/mm grating), which is connected to a liquid-nitrogencooled back-illuminated charge-coupled-device (CCD) camera. The spectral resolution of this system is a $0.06 \mathrm{~nm}$ wavelength, which is equivalent to an energy resolution of 250 $\mu \mathrm{eV}$ in the spectral range investigated. We scan the uncoated

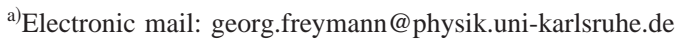

fiber tip in a constant height of $100 \mathrm{~nm}$ above the surface, i.e., the feedback loop is inactive. Under these conditions, the measured spatial resolution is $200 \mathrm{~nm}$. The data sets shown in the following are always based on 1600 individual spectra, each with $0.5 \mathrm{~s}$ exposure time of the CCD camera, taken in a $2 \mu \mathrm{m} \times 2 \mu \mathrm{m}$ area with $50 \mathrm{~nm}$ separation between adjacent points.

Four examples of individual photoluminescence spectra at a sample temperature of $T=20 \mathrm{~K}$ from four different samples, A, B, C, and D, are shown in Fig. 1. Sample A stems from the Ioffe institute in St. Petersburg and is grown by molecular-beam epitaxy (MBE). ${ }^{11}$ The nominal average CdSe layer thickness is $3.6 \mathrm{ML}$, the top ZnSe layer is $10 \mathrm{~nm}$ thin, which is suitable for optical near-field experiments. Samples B, C, and D are grown by mixed elemental and compound-source MBE in-house. ${ }^{12}$ The nominal average CdSe layer thickness of sample B is $2 \mathrm{ML}$, the top $\mathrm{ZnSe}$ layer is $20 \mathrm{~nm}$ thin. Samples C (2.3 ML, $20 \mathrm{~nm} \mathrm{ZnSe)}$ and D ( $3 \mathrm{ML}, 45 \mathrm{~nm} \mathrm{ZnSe}$ ) are thermally annealed at $320^{\circ} \mathrm{C}$ directly after $\mathrm{CdSe}$ deposition (i.e., prior to cap growth) for a duration of 5 and $10 \mathrm{~min}$, respectively. This is to initiate Oswald ripening of $\mathrm{CdSe}$ islands. Obviously, the individual photoluminescence spectra of these samples look very differ-

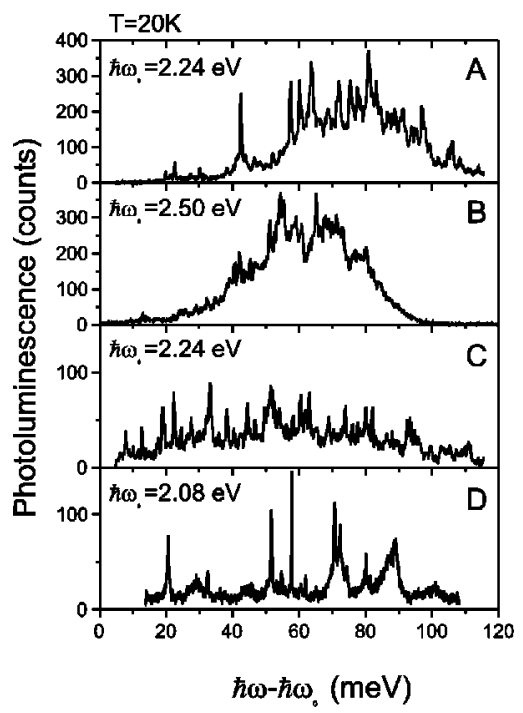

FIG. 1. Individual photoluminescence spectra of samples A, B, C, and D at a sample temperature of $20 \mathrm{~K}$. For clarity, the spectra are shown on the same scale. 
ent in terms of line density and width of the line distribution. Also, note the large shift in the overall spectral position $\hbar \omega_{0}$.

Back to our initial question: What can be learned from such spectra? We first tried to apply the idea of Refs. 7 and 8 directly. This leads to difficulties, which can already be anticipated from the spectra shown in Fig. 1. Beside the many resolved lines, one also has a broad background in each individual spectrum. The autocorrelation of this undesired contribution is so prominent that it overwhelms all other features by far (not shown). Consequently, we eliminate this background by calculating the average and subtract this average from each individual spectrum of this set. In addition to this, we normalize each individual photoluminescence spectrum to equal the integral before starting the above procedure. For photoluminescence spectra $I(\hbar \omega)$, which consist of a series of sharp lines, i.e., $I(\hbar \omega)=\Sigma_{n} I_{n} \delta\left(\hbar \omega-\hbar \omega_{n}\right)$ with arbitrary values of $I_{n}$, one can show that $\int_{0}^{\infty}\langle C(\Delta E)\rangle d(\Delta E)=0$ holds under these conditions. We summarize the complete procedure: (1) normalization of the individual spectra to equal the spectral integral; (2) subtraction of the average of these normalized spectra, $\delta I_{n}(\hbar \omega)$ $=I_{n}(\hbar \omega)-\left\langle I_{n}(\hbar \omega)\right\rangle$; (3) calculation of the individual autocorrelations, $C(\Delta E)=\int \delta I_{n}\left(\hbar \omega^{\prime}\right) \delta I_{n}\left(\hbar \omega^{\prime}+\Delta E\right) \mathrm{d} \omega^{\prime} ; \quad$ (4) averaging over all individual autocorrelations; and (5) normalization of the maximum of $\langle C(\Delta E)\rangle$ at $\Delta E=0$ to unity. Figure 2 illustrates this procedure for two examples: A set of measured spectra of sample A (left column) and a set of computer-generated random spectra (right column). In these random spectra we have chosen the number of lines, their width, and their (Gaussian) distribution to roughly match both the individual measured spectra and their average. All data sets are treated by the same program. For the computergenerated spectra, we find a maximum at $\Delta E=0$ and no further structure. The same behavior is found in many other computer-generated sets with different numbers of lines and/or different distributions (not shown). This result is expected and illustrates that the defined procedure does not lead to artifacts. In contrast to the computer-generated random spectra, the experimental data show a correlation peak around $\Delta E=18 \mathrm{meV}$ [arrow in Fig. 2(e)].

Amazingly, this correlation is very similar for many different measurements on samples A, B, C, and D-although the individual spectra (see Fig. 1) look considerably different. This is shown in Fig. 3, where, for each sample, we depict two data sets (Nos. 1 and 2) from different areas on the sample. Obviously, the correlations in the photoluminescence lines of these $\mathrm{CdSe} / \mathrm{ZnSe}$ layers are generic for this particular material system.

These correlations are consistent with spectra which consist of sets of pairs of lines. For each pair the energy spacing has to be around $18 \mathrm{meV}$. Can we learn more about the relative strength of the two lines in the pairs? Following Ref. 13, one can multiply the individual photoluminescence spectra with filter functions $f(\hbar \omega)$ [before performing step (1)]. Exponential functions, i.e., $f(\hbar \omega) \propto \exp \left(\hbar \omega / E_{0}\right)$, are especially simple to interpret. For the case of $E_{0}>0$ and for spectra which consist of pairs of lines with one main line and a weaker high-energy sideband, the sideband would be enhanced by a certain factor (independent on its absolute ener-
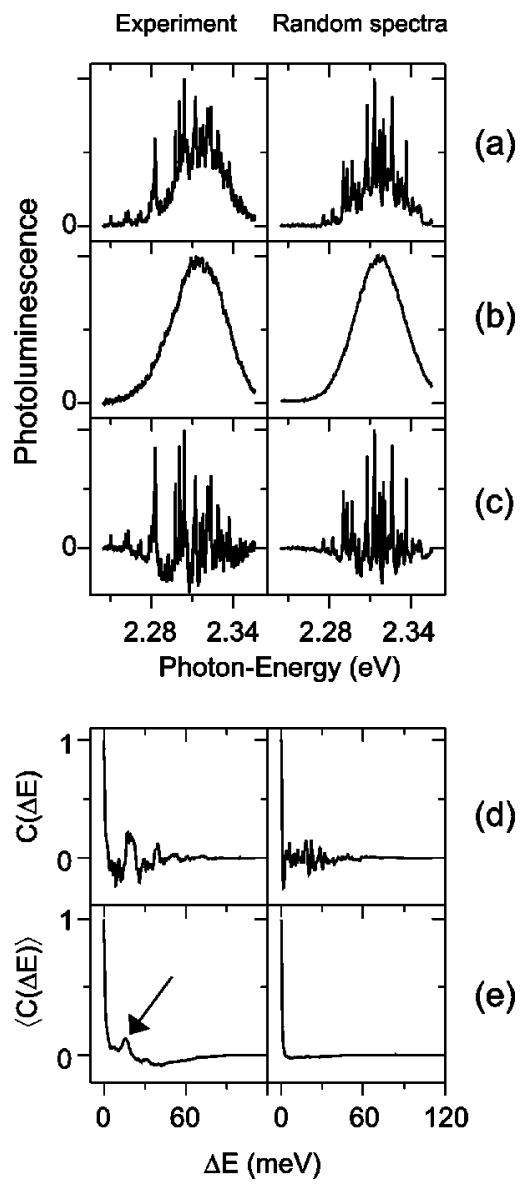

FIG. 2. Illustration of the statistical procedure for experimental data on sample A (left column) and for computer-generated random spectra (right column). (a) Individual spectra, normalized to equal spectral integral; (b) average; $(c)=(a)-(b)$; (d) autocorrelation of (c); and (e) averaged autocorrelation $\langle C(\Delta E)\rangle$ (normalized to unity).

getic position). Consequently, the correlation function $\langle C(\Delta E)\rangle$ would exhibit enhanced correlations. Similarly, a low-energy sideband would be suppressed for $E_{0}>0$ and vice versa for $E_{0}<0$. This idea has been verified explicitly by numerical simulation. Applying this procedure to the data

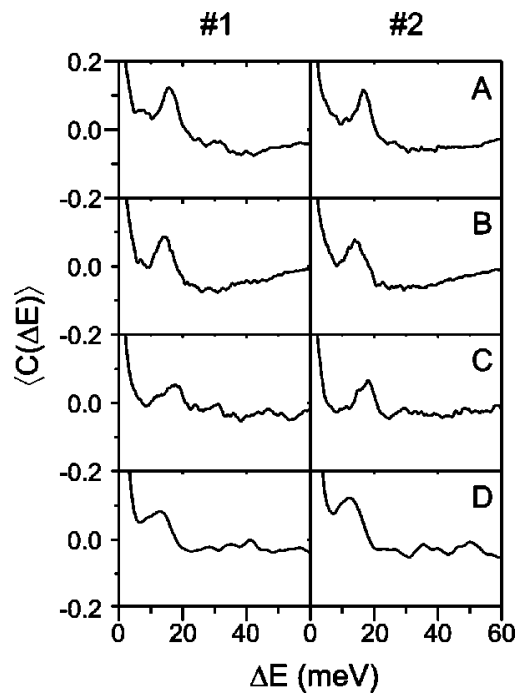

FIG. 3. Correlation function $\langle C(\Delta E)\rangle$ of two areas (Nos. 1 and 2) on each of the samples A, B, C, and D. Note that the position of the correlation peak around 15-20 meV does not vary much.

AlP license or copyright; see http://apl.aip.org/apl/copyright.jsp 


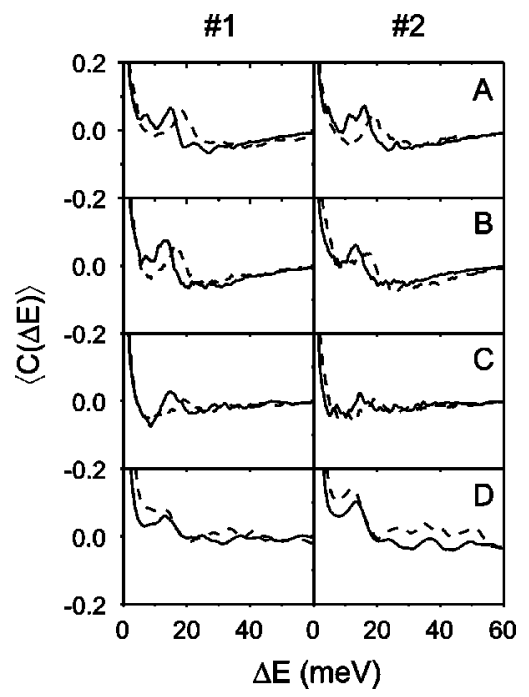

FIG. 4. Correlation function $\langle C(\Delta E)\rangle$ of the same two areas (Nos. 1 and 2) as in Fig. 3. Filters with $E_{0}=+18 \mathrm{meV}$ (full lines) and $E_{0}=-18 \mathrm{meV}$ (dashed lines). Note that the height of the correlation peak does not change much.

shown in Fig. 3, leads to the curves shown in Fig. 4 (full lines correspond to $E_{0}=+18 \mathrm{meV}$, dashed lines to $E_{0}$ $=-18 \mathrm{meV}$ ). Obviously, the determined height of the correlation peak is roughly the same for $E_{0}= \pm 18 \mathrm{meV}$. This implies that we have pairs of lines with comparable strength - on the average. This can either mean that the two lines in the pairs have identical strength in each case or that they strongly fluctuate independently. By additional simulations we have found that the latter scenario would lead to a correlation peak in $\langle C(\Delta E)\rangle$, which is definitely too weak to be consistent with the experiment. For lines of identical strength, our simulations give results which are consistent with the experiment (Fig. 3).

We have already concluded that our experimental results are consistent with individual spectra which consist of sets of pairs of lines. The last section has shown that in each pair the two lines must have comparable strength. This makes interpretations along the lines of a phonon sideband quite unlikely (the LO-phonon energy of bulk CdSe is $26 \mathrm{meV}$, that of $\mathrm{ZnSe}$ is $32 \mathrm{meV}$ ). Phonon sidebands are usually weak. Islands of rather uniform size ("self-organized quantum dots") would lead to a rather uniform level spacing. Several researchers ${ }^{3,14}$ have indeed reported islands in this material system with an areal density around $10^{11} \mathrm{~cm}^{-2}$ and with sizes of 5-10 nm. The internal motion of the electron and the hole within the exciton would lead to a further smearing out, hence to a broadening, of the potential for the excitons ${ }^{8}$ (for bulk CdSe, the exciton Bohr radius is $9 \mathrm{~nm}$ ). The expected level spacing for such effective potential wells seems consis- tent with the correlation energy of $18 \mathrm{meV}$ observed in our work (the exciton mass of bulk CdSe is $1.3 \times m_{0}$, that of ZnSe $\left.1.9 \times m_{0}\right)$. Also, the number of lines in each spectrum is roughly consistent with an areal density of islands of $10^{11} \mathrm{~cm}^{-2}$. If this interpretation concerning sets of pairs of lines from rather uniform islands is correct indeed, our observations would be evidence for a highly nonthermal distribution of excitons in these islands (due to the "phonon bottleneck"). Biexcitonic effects in these dots could also lead to the observed positive correlations. While we have not found any clear intensity dependence when attenuating the incident laser intensity from $200 \mathrm{nW}$ down to $20 \mathrm{nW}$, it is interesting to note that the quantum-dot biexciton binding energy of $\approx 20 \mathrm{meV}$ observed in single-dot experiments ${ }^{15}$ is close to the numbers seen here. This would imply a rather uniform biexciton binding energy in the quantum dots.

Note added in proof: Additional recent experiments have shown that the positive correlation peak remains unchanged for incident laser intensities even down to $2 \mathrm{nW}$. Also, it remains unchanged for temperatures in the range of 4.2 to $80 \mathrm{~K}$.

This research has been supported by the DFG-SFB 195, the DFG-GK 284, and is performed within the Institut für Nanotechnologie der Universität Karlsruhe (TH). The research of one of the authors (M.W.) is funded by the DFG Leibniz price. The authors thank E. Runge, R. Zimmermann, and Th. Schimmel for stimulating discussions.

${ }^{1}$ H. F. Hess, E. Betzig, T. D. Harris, L. N. Pfeiffer, and K. W. West, Science 264, 822 (1994).

${ }^{2}$ A. Zrenner, L. V. Butov, M. Hagn, G. Abstreiter, G. Böhm, and G. Weimann, Phys. Rev. Lett. 72, 3382 (1994).

${ }^{3}$ F. Flack, N. Samarth, V. Nikitin, P. A. Crowell, J. Shi, J. Levy, and D. Awschalom, Phys. Rev. B 54, 17312 (1996).

${ }^{4}$ U. Jahn, M. Ramsteiner, R. Hey, H. T. Grahn, E. Runge, and R. Zimmermann, Phys. Rev. B 56, R4387 (1997).

${ }^{5}$ L. M. Robinson, H. Rho, J. C. Kim, H. E. Jackson, L. M. Smith, S. Lee, M. Dobrowolska, and J. K. Furdyna, Phys. Rev. Lett. 83, 2797 (1999)

${ }^{6}$ Q. Wu, R. D. Grober, D. Gammon, and D. S. Katzer, Phys. Rev. Lett. 83, 2652 (1999).

${ }^{7}$ E. Runge and R. Zimmermann, Phys. Status Solidi B 206, 167 (1998).

${ }^{8}$ E. Runge and R. Zimmermann, Ann. Phys. (Leipzig) 8, 229 (1999).

${ }^{9}$ V. Savona, S. Haacke, and B. Deveaud, Phys. Rev. Lett. 84, 183 (2000).

${ }^{10} \mathrm{Ch}$. Adelmann, J. Hetzler, G. Scheiber, Th. Schimmel, M. Wegener, H. B. Weber, and H. v. Löhneysen, Appl. Phys. Lett. 74, 179 (1999).

${ }^{11}$ S. V. Ivanov, A. A. Toropov, T. V. Shubina, S. V. Sorokin, A. V. Lebedev, I. V. Sedova, P. S. Kop'ev, G. R. Pozina, J. P. Bergmann, and B. Monemar, J. Appl. Phys. 83, 3168 (1998).

${ }^{12}$ E. Kurtz, M. Schmidt, M. Baldauf, S. Wachter, M. Grün, D. Litvinov, S. K. Hong, J. X. Shen, T. Yao, D. Gerthsen, H. Kalt, and C. Klingshirn, J. Cryst. Growth 214-215, 712 (2000).

${ }^{13} \mathrm{E}$. Runge (private communications).

${ }^{14}$ N. Peranio, A. Rosenauer, D. Gerthsen, S. V. Sorokin, I. V. Sedova, and S. V. Ivanov (unpublished).

${ }^{15}$ G. Bacher, R. Weigand, J. Seufert, V. D. Kulakovskii, N. A. Gippius, and A. Forchel, Phys. Rev. Lett. 83, 4417 (1999). 